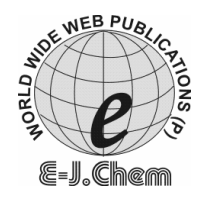

E-Journal of Chemistry

http://www.e-journals.net

2009, 6(1), 13-22

\title{
Analysis of Heavy Metals Concentration in Soil and Litchens from Various Localities of Hosur Road, Bangalore, India
}

\author{
ABIDA BEGUM ${ }^{*}$, M. RAMAIAH ${ }^{\S}$, HARIKRISHNA $^{\#}$, \\ IRFANULLA KHAN ${ }^{\text {II }}$ and K.VEENA. \\ *Department of Chemistry, \\ P. E. S. School of Engineering, Bangalore, India. \\ ${ }^{\S}$ NMKRV Degree College, " Shirdi Sai Engg. College, \\ ${ }^{\mathbb{I}}$ Mohammed Ishaq College and Maharani's Science College, Bangalore, India. \\ drabid.drabida@gmail.com
}

Received 29 March 2008; Accepted 20 May 2008

\begin{abstract}
Assessment of heavy metal content in litchens and soil samples from various localities of Hosur Road, Bangalore south was undertaken. Topsoil samples $(0-10 \mathrm{~cm})$ were taken at various locations, the metals analysed were $\mathrm{Cr}$, $\mathrm{Pb}, \mathrm{Fe}, \mathrm{Zn}, \mathrm{Ni}$ and $\mathrm{Cu}$. The geoaccumulation index of these metals in the soils under study residential areas indicated that they are uncontaminated with $\mathrm{Ni}, \mathrm{Zn}$, and $\mathrm{Fe}$ and moderately contaminated with $\mathrm{Cr}$ and $\mathrm{Pb}$. In Industrial areas and traffic junctions the concentration of $\mathrm{Fe}, \mathrm{Pb}$ and $\mathrm{Ni}$ was maximum. Heavy metal accumulation in few prominent lichens of some localities was analysed. $\mathrm{Cr}$ and $\mathrm{Pb}$ were maximum in Chrysothrix candelaris (L.) Laundon, at the gardens of Madiwala and Silk Board junction with 95.29 and $623.95 \mu \mathrm{g} \mathrm{g}^{-1}$ dry weight respectively. $\mathrm{Fe}$ and $\mathrm{Cu}$ were maximum in Bulbothrix isidiza (Nyl.). Hale and Pyxine petricola $\mathrm{Nyl}$ at Central Prison campus and Kendriya Sadan campus with 22721 and $338.12 \mu \mathrm{g} \mathrm{g}^{-1}$ dry weight respectively, Lecanora perplexa Brodo at Infosis and Wipro Campus, electronic city have 531.5 and $634 \mu \mathrm{g} \mathrm{g}^{-1}$ dry weight of $\mathrm{Zn}$. While Ni and Fe were maximum in Arthopyreniaceae at Shanti Niketan of MICO Limited with 1100 and $23200 \mu \mathrm{g} \mathrm{g}^{-1}$ dry weight respectively.
\end{abstract}

Keywords: Heavy metals, Soils, Litchen species, Hosur road, Bangalore south.

\section{Introduction}

Bangalore, a fast growing metropolis, the garden city is the capital of the Southern Indian State of Karnataka situated at about 1000 meters above sea level, it is known for its 
salubrious climate where temperatures remain moderate through out the year. The maximum temperature in July is around $32^{\circ} \mathrm{C}$ and the minimum around $22^{\circ} \mathrm{C}$. It is connected by air, rail and road to all major cities of the country and has direct international connections to many cities worldwide. Hosur Road is the part of National Highway which connects the city of Bangalore and the Tamil Nadu border town of Hosur. It is a four-lane highway which also has service lanes on either side at the busier parts. Apart from being a part of the National Highway, the road is also significant because it houses many industrial and IT business houses. The famous IT industrial park Electronics City is also located in Hosur Road. Owing to the large number of offices and its use as major truck route, the road is chocked during most part of the day. In recent years, the main highway connecting Electronics City with Bangalore has seen a large increase in vehicular traffic, causing traffic jams at several road junctions. Currently, major infrastructure improvements are taking place including widening of the road and the building of a 9 kilometer elevated highway. It promises to reduce and redistribute the traffic for a smoother flow. The highway, Hosur road also has some buildings of some major IT players like BOSCH, Sasken, Convergys etc, along with showrooms of major auto manufacturers like Toyota etc. Air pollution in Bangalore city is undoubtedly the exhaust emission from vehicular traffic ${ }^{1}$. Inadequate public transport system has led to an increase in the use of personalized vehicles, Congested traffic, poor road conditions and outdated automotive technology add to the increase in vehicular emissions. Large quantities of pollutants have continuously been introduced into ecosystems as a consequence of urbanization and industrial processes. Metals are persistent pollutants that can be biomagnified in the food chains, becoming increasingly dangerous to human and wildlife This has led to the development of monitoring schemes aimed at directly measuring levels of contaminants in various organisms, and biomonitoring schemes that use indicator species to estimate the levels in other parts of the ecosystem ${ }^{2}$. Therefore, assessing pollutants in different components of the ecosystem has become an important task in preventing risk to natural life and public health Heavy metals enter into the environment mainly via three routes: (i) deposition of atmospheric particulate, (ii) disposal of metal enriched sewage sludges and sewage effluents and (iii) by-products from metal mining process. Soil is one of the repositories for anthropogenic wastes. Biochemical processes can mobilize them to pollute water supplies and impact food chains. Trace heavy metal contamination in the soils is a major concern because of their toxicity and threat to human life and the environment ${ }^{3}$. Heavy metals such as $\mathrm{Cu}, \mathrm{Cr}, \mathrm{Cd}, \mathrm{Ni}$, and $\mathrm{Pb}$ are potential soil and water pollutants. Heavy metals studies have been conducted in soils with differing levels of anthropogenic influences such as in highly populated and industrialized cities. Most heavy metal concentration in urban cities has insufficient drainage system leading to contamination of immediate surroundings and the ground water with trace heavy metals. Heavy metal studies are necessary to evaluate both soil/sediment and groundwater contamination. Also most of the municipal water supplies come from boreholes, most of which are shallow wells. Urban food security in India is a matter of growing concern. The problem of environmental pollution due to toxic metals has begun to cause concern now in most major metropolitan cities. The toxic heavy metals entering the ecosystem may lead to geoaccumulation, bioaccumulation and biomagnifications. Heavy metals like $\mathrm{Fe}, \mathrm{Cu}$, $\mathrm{Zn}, \mathrm{Ni}$ and other trace elements are important for proper functioning of biological systems and their deficiency or excess could lead to a number of disorders. Food chain contamination by heavy metals has become a burning issue in recent years because of their potential accumulation in biosystems through contaminated water, soil and air ${ }^{4}$.

Lichens are a group of symbiotic organisms exploiting variety of habitats throughout the world. Lichens have long been recognized as sensitive indicators of environmental conditions. Lichens show their sensitivity to air pollution in various ways such as decline in diversity, absence of sensitive species, and morphological, anatomical and physiological 
changes. They are also proved to be good accumulators of many elements, particularly heavy metals. Various heavy metals such as $\mathrm{Pb}, \mathrm{Zn}, \mathrm{Ni}, \mathrm{Cu}, \mathrm{Hg}$ and $\mathrm{Cr}$, considered as toxic for many other living organisms. The purpose of the present study is to provide information regarding lichen flora of certain localities of Hosur Road. Together with the accumulation of six heavy metals in some commonly growing lichen species of the area. The major cause is the emission from industrial establishment, traffic snarls, biodegradation and uncontrolled burning of garbage accumulated in dumping yards contribute to urban air pollution. The impact of pollution in the vicinity of overcrowded cities and from industrial effluents and automobile exhausts has reached a disturbing magnitude and is arousing public awareness ${ }^{7,8}$.

\section{Data collection and analysis}

15 Soil samples (three replicates) were collected at surface level $(0-10 \mathrm{~cm}$ in depth) were collected from various locations to cover industrial, commercial and residential areas. The collected soil samples were air-dried and sieved into coarse and fine fractions. Well-mixed samples of $2 \mathrm{~g}$ each were taken in $250 \mathrm{~mL}$ glass beakers and digested with $8 \mathrm{~mL}$ of aqua regia on a sand bath for 2 hours ${ }^{5}$. After evaporation to near dryness, the samples were dissolved with $10 \mathrm{~mL}$ of $2 \%$ nitric acid, filtered and then diluted to $50 \mathrm{~mL}$ with distilled water. Heavy metal concentrations of each fraction was analysed by Atomic Absorption Spectrophotometry using GBC Avanta version 1.31 by flame Atomization ${ }^{6}$. Quality assurance was guaranteed through double determinations and use of blanks for correction of background and other sources of error. EC of the soil samples were determined from saturation extract by conductivity meter. Measurement of $\mathrm{pH}$ of the water and soil samples were done (soil and water ratio 1:25) were done with help of a glass electrode $\mathrm{pH}$ meter ${ }^{5,6}$.

Lichens from all the available tree species were collected from 15 localities in Hosur Road (Table 1) during September and October 2007. The lichens were carefully removed from the bark snapper blade and were oven-dried to a constant weight ${ }^{7,8}$ at $80^{\circ} \mathrm{C}$. The dried lichen samples (three replicates) were then powdered $(0.5 \mathrm{~g})$ for further metal analysis. Lichen samples were extracted and analysed with (aqua regia) a mixture of concentrated $\mathrm{HCl}$ and $\mathrm{HNO}_{3}(3: 1)$ and digested to $80^{\circ} \mathrm{C}$, few drops of perchloric acid is added. The mixture was filtered through Whatman filter paper No. 42. The filtrate was diluted to the desired volume with deionised water. The total concentrations of $\mathrm{Cr}, \mathrm{Pb}, \mathrm{Fe}, \mathrm{Zn}, \mathrm{Ni}$ and $\mathrm{Cu}$ in the filtrate were determined by atomic adsorption spectrometer Different concentrations of the air-dried Soil Samples dissolved in distilled water were analyzed for $\mathrm{pH}$, alkalinity, $\mathrm{CO}_{2}$ and dissolved oxygen.

\section{Results and Discussion}

The heavy metal analysis of (Table 2) various species of lichens collected, analysed and identified (Botonically from the department of Botony SJR Women's Degree College Rajajinagar, Bangalore.) in the 12 localities of Hosur Road is reported. Cr concentration ranged between 2.50-95.29 $\mu \mathrm{g} \mathrm{g}^{-1}$ dry weights of litchens species. Maximum concentration was in Chennakeshavanagar Main Road 95.29 Bomannahalli traffic junction 34.57 Begur Road $36.43 \mu \mathrm{g} \mathrm{g}^{-1}$ dry weight. Pb concentration ranged between 31.92-623.95 $\mu \mathrm{g} \mathrm{g}^{-1}$ dry weight of litchens species. Maximum concetration was in Bomannahalli traffic junction 623.95. Mangamanapalya Road 484.69 and Agara Lake $203.10 \mu \mathrm{g} \mathrm{g}^{-1}$ dry weight. Fe concentration ranged between 55.47-9795 $\mathrm{g} \mathrm{g} \mathrm{g}^{-1}$ dry weights of litchens species. Maximum concentration was in Madiwala Market $9795 \mu^{g} \mathrm{~g}^{-1}$ dry weight. Chennakeshavanagar Main $9202 \mu \mathrm{g} \mathrm{g}^{-1}$ dry weight $\mathrm{Zn}$ concentration ranged between $79.86-831.01 \mu \mathrm{g} \mathrm{g}^{-1}$ dry weights of 
litchens species. Maximum concentration was in Begur Road $831.01 \mu \mathrm{g} \mathrm{g}^{-1}$ dry weight, Bomannahalli traffic junction $553.92 \mu \mathrm{g} \mathrm{g}^{-1}$ dry weight. Agara Lake $384.55 \mu \mathrm{g} \mathrm{g}^{-1}$ dry weight. $\mathrm{Cu}$ concentration ranged between 5.02-338.12 $\mathrm{g} \mathrm{g} \mathrm{g}^{-1}$ dry weights of litchens species. Maximum concentration was in Shanthi Niketan gardens MICO Naganathapura plant 338.12 $\mu \mathrm{g} \mathrm{g}^{-1}$ dry weight, Bomannahalli traffic junction $158.32 \mu \mathrm{g} \mathrm{g}^{-1}$ dry weight. Chennakeshavanagar Main Road $115.19 \mu \mathrm{g} \mathrm{g}^{-1}$ dry weight. Ni concentration ranged between 343-1409.34 $\mu \mathrm{g} \mathrm{g}^{-1}$ dry weights of litchens species. Maximum concentration was in Shanthi Niketan gardens MICO Naganathapura plant 1409.34, Central prison Road 843.10 and Chennakeshavanagar Main Road $800.7 \mu \mathrm{g} \mathrm{g}^{-1}$ dry weight. Respectively (Table 2) It is observed that maximum heavy metal pollution near the industrial, traffic junction where traffic jams and the legendary 'go-slow' of automobiles is the order of the day and in localities of large population concentration and relatively small areas under poor conditions of sanitation ${ }^{10-12}$.

Table 1. Some physicochemical parameters of the soil samples.

\begin{tabular}{|c|c|c|c|c|c|}
\hline Sample Stations & $\mathrm{pH}$ & $\begin{array}{c}\mathrm{EC} \\
\mathrm{ds} / \mathrm{cm}\end{array}$ & $\begin{array}{c}\text { Dissolved } \\
\text { Oxygen } \mathrm{mg} / \mathrm{L}\end{array}$ & $\begin{array}{l}\text { Carbon dioxide } \\
\mathrm{mg} / \mathrm{L}\end{array}$ & $\begin{array}{l}\text { Alkalinity } \\
\mathrm{mg} / \mathrm{L}\end{array}$ \\
\hline $\begin{array}{l}\text { Kendriya Sadan } \\
\text { Koramangala }\end{array}$ & 7.46 & 7.56 & 7.36 & 5.62 & 19.36 \\
\hline Agara Lake & 6.20 & 4.88 & 7.60 & 5.79 & 19.22 \\
\hline Madiwala Market & 7.30 & 7.05 & 6.60 & 5.86 & 19.18 \\
\hline $\begin{array}{l}\text { Ayappa Swamy } \\
\text { Temple Road }\end{array}$ & 6.67 & 5.75 & 6.78 & 5.32 & 19.45 \\
\hline $\begin{array}{l}\text { Madiwala Police } \\
\text { Station Junction }\end{array}$ & 7.55 & 29.74 & 6.99 & 5.60 & 19.45 \\
\hline $\begin{array}{l}\text { Bomannahalli Trraffic } \\
\text { Junction }\end{array}$ & 6.25 & 22.23 & 7.27 & 5.01 & 19.33 \\
\hline Begur Road & 6.55 & 28.90 & 5.29 & 5.01 & 17.43 \\
\hline $\begin{array}{l}\text { Mangamanapalya } \\
\text { Road }\end{array}$ & 6.55 & 10.83 & 5.36 & 5.62 & 19.55 \\
\hline $\begin{array}{l}\text { B.D.A Complex H.S.R } \\
\text { Layout }\end{array}$ & 7.94 & 5.80 & 7.12 & 5.88 & 19.21 \\
\hline $\begin{array}{l}\text { Chennakeshavanagar } \\
\text { Main Road }\end{array}$ & 7.48 & 9.66 & 7.12 & 5.79 & 19.78 \\
\hline $\begin{array}{l}\text { Shanthi Niketan } \\
\text { gardens MICO }\end{array}$ & 7.30 & 7.05 & 7.386 & 5.81 & 19.24 \\
\hline Central prison Road & 7.54 & 4.85 & 7.56 & 5.62 & 19.56 \\
\hline $\begin{array}{l}\text { Infosys garden } \\
\text { Electronic City }\end{array}$ & 7.65 & 7.65 & 6.68 & 5.62 & 19.43 \\
\hline $\begin{array}{l}\text { Wipro Garden } \\
\text { Electronic City }\end{array}$ & 7.35 & 5.75 & 6.68 & 5.45 & 17.68 \\
\hline $\begin{array}{l}\text { Electronic city Phase } 1 \\
\text { Junction. }\end{array}$ & 6.89 & 9.37 & 6.79 & 5.34 & 17.48 \\
\hline
\end{tabular}




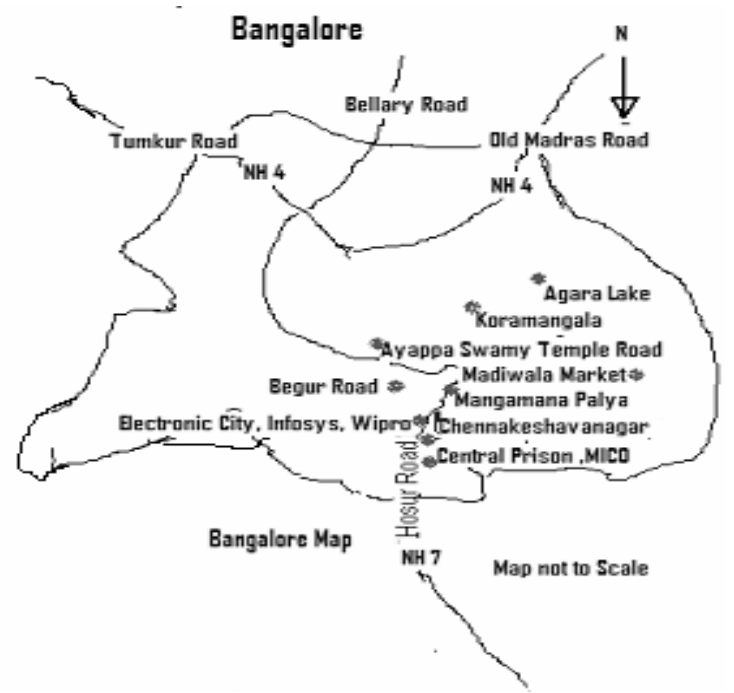

Figure 1. Study stations of Hosur Road
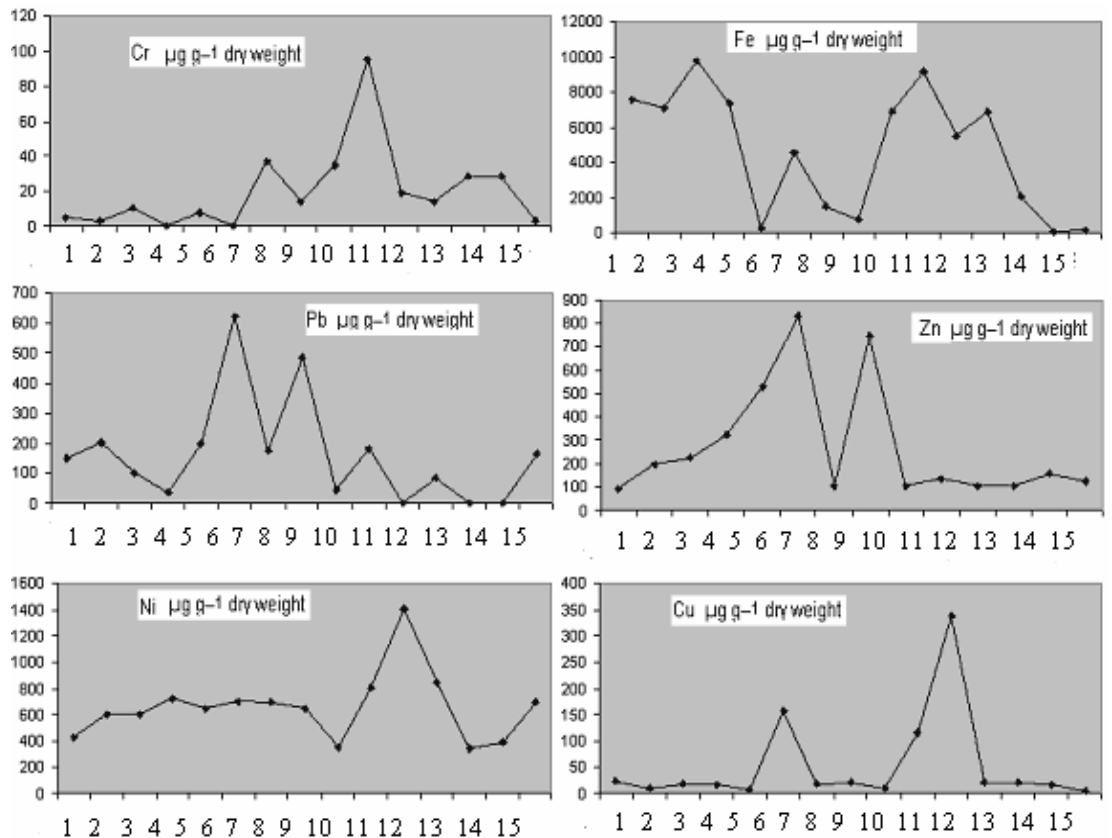

Figure 2. Heavy metal concentration $\mu \mathrm{g} \mathrm{g}^{-1}$ dry weight in litchens species at various localities* in Hosur Road.

*(Kendriya Sadan Koramangala, Agara Lake , Madiwala Market , Madiwala Market , Ayappa Swamy Temple Road, Madiwala Police Station Junction, Bomannahalli Junction Begur Road, Mangamanapalya Road, B.D.A Complex H.S.R Layout, Chennakeshavanagar Main Road, Shanthi Niketan gardens MICO Naganathapura plant, Central prison Road, Infosys garden Electronic City, wipro Garden Electronic City, Electronic city Phase 1 Junction.) 

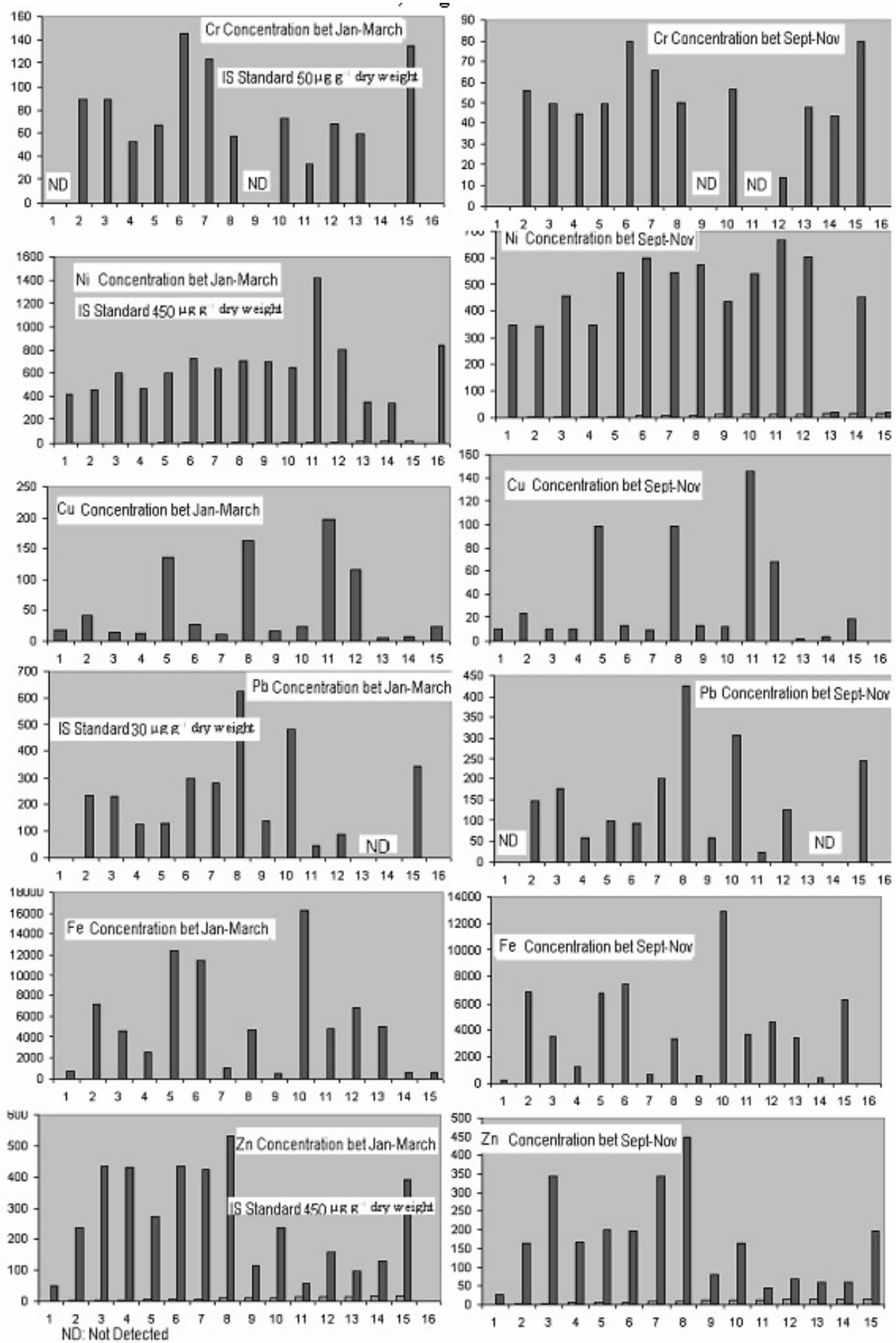

Figure 3. Heavy metal concentration $\mu \mathrm{g} \mathrm{g}^{-1}$ dry weight in soils of Hosur Road, Bangalore south. 
Table 2. Litchen species found in various localities of Hosur Road, Bangalore south.

\begin{tabular}{|c|c|c|c|}
\hline $\begin{array}{l}\text { S. } \\
\text { No. }\end{array}$ & Litchen Species & $\begin{array}{l}\text { Growth } \\
\text { form }\end{array}$ & Stations \\
\hline 1 & $\begin{array}{l}\text { C. candelaris (L.) Laundon } \\
\text { L. perplexa Brodo } \\
\text { D. aegialita (Afz. in Ach.) Moore } \\
\text { D. consimilis (Stirton) Awasthi } \\
\text { L. perplexa Brodo }\end{array}$ & $\begin{array}{l}\mathrm{C} \\
\mathrm{F} \\
\mathrm{C} \\
\mathrm{F} \\
\mathrm{F}\end{array}$ & Kendriya Sadan Koramangala \\
\hline 2 & $\begin{array}{l}\text { G. scripta (L.) Ach. } \\
\text { L. perplexa Brodo } \\
\text { D. aegialita (Afz. in Ach.) Moore }\end{array}$ & $\begin{array}{l}\mathrm{F} \\
\mathrm{F} \\
\mathrm{C}\end{array}$ & Agara Lake \\
\hline 3 & leucosorodes $\mathrm{Nyl}$. & $\mathrm{F}$ & Madiwala market \\
\hline 4 & P. cocoes (Swartz) Nyl. & $\mathrm{C}$ & Ayappa Swamy Temple Road \\
\hline 5 & D. aegialita (Afz. in Ach.) Moore & $\mathrm{C}$ & $\begin{array}{c}\text { Madiwala Police Station } \\
\text { Junction }\end{array}$ \\
\hline 6 & L. perplexa Brodo & $\mathrm{F}$ & Bomannahalli Junction \\
\hline 7 & P. austrosinensis (Zahlbr.) Hale & $\mathrm{C}$ & Begur Road \\
\hline 8 & P. nanospora (A. Singh) Upreti & $\mathrm{F}$ & Mangamanapalya Road \\
\hline 9 & $\begin{array}{l}\text { C. candelaris }(\mathrm{L} .) \text { Laundon } \\
\text { L. perplexa Brodo }\end{array}$ & $\begin{array}{l}\mathrm{C} \\
\mathrm{F}\end{array}$ & B.D.A Complex H.S.R Layout \\
\hline 10 & $\begin{array}{l}\text { D. aegialita (Afz. in Ach.) Moore } \\
\text { L. perplexa Brodo }\end{array}$ & $\begin{array}{l}\mathrm{C} \\
\mathrm{F}\end{array}$ & $\begin{array}{c}\text { Chennakeshavanagar Main } \\
\text { Road }\end{array}$ \\
\hline 11 & $\begin{array}{l}\text { P. petricola } \text { Nyl. in Crombie } \\
\text { L. perplexa Brodo } \\
\text { D. aegialita (Afz. in Ach.) Moore }\end{array}$ & $\begin{array}{l}\mathrm{C} \\
\mathrm{F} \\
\mathrm{C}\end{array}$ & $\begin{array}{l}\text { Shanthi Niketan gardens } \\
\text { MICO Naganathapura plant }\end{array}$ \\
\hline $\begin{array}{l}12 \\
13\end{array}$ & $\begin{array}{l}\text { C. candelaris }(\mathrm{L} .) \text { Laundon } \\
\text { C. candelaris }(\mathrm{L} .) \\
\text { Laundon } \\
\text { L. perplexa Brodo }\end{array}$ & $\begin{array}{l}\mathrm{C} \\
\mathrm{C} \\
\mathrm{F} \\
\mathrm{F}\end{array}$ & $\begin{array}{c}\text { Central Prison Road } \\
\text { Infosys Garden Electronic } \\
\text { City }\end{array}$ \\
\hline 14 & $\begin{array}{l}\text { P. praesorediosa }(\mathrm{Nyl} \text {.) Hale } \\
\text { L. perplexa Brodo }\end{array}$ & $\begin{array}{l}\mathrm{F} \\
\mathrm{F}\end{array}$ & Wipro Garden Electronic City \\
\hline 15 & $\begin{array}{l}P . \text { concinna Erichsen } \\
P \text {. petricola Nyl. in Crombie }\end{array}$ & $\begin{array}{l}\mathrm{C} \\
\mathrm{C} \\
\end{array}$ & $\begin{array}{l}\text { Electronic City Phase } 1 \\
\text { Junction. }\end{array}$ \\
\hline
\end{tabular}

C: Crustose; F: Foliose.

Table $3 \& 4$ shows the results of heavy metal analysis in soils. Metal concentrations in soils were minimum during August to October 2007 and maximum during January-March 2007. Low values may be explained due to leaching effect, and degree of flushing during heavy rains. Heavy metal concentrations were minimum in stations $1,9,11$ (Ni concentration was maximum), 13 and 14 as these localities are well maintained with all amenities. Maximum concentration of all analysed heavy metals was observed in all traffic junction particularly in Bomanahalli traffic junction which is serious and matter of concern. Maximum heavy metal concentration was found in Agara lake and also thickly populated industrial areas like Begur Road and Mangamanapalya Road where sanitation and sewage maintenance facility is very poor. 
Table 3. Heavy metal concentration $/ \mu \mathrm{g} \mathrm{g}^{-1}$ dry weight in selected litchens species.

\begin{tabular}{|c|c|c|c|c|c|c|c|}
\hline $\begin{array}{l}\text { S. } \\
\text { No. }\end{array}$ & Stations and Lichen Species & $\mathrm{Cr}$ & $\mathrm{Pb}$ & $\mathrm{Fe}$ & $\mathrm{Zn}$ & $\mathrm{Cu}$ & $\mathrm{Ni}$ \\
\hline \multirow[t]{2}{*}{1} & $\begin{array}{l}\text { Kendriya Sadan Koramangala } \\
\text { C. candelaris (L.) Laundon }\end{array}$ & 5.18 & ND & 7556 & 95.76 & 23.72 & 420.90 \\
\hline & D. consimilis (Stirton) Awasthi & ND & 31.92 & 7081 & 198.14 & 22.22 & 453.70 \\
\hline \multirow[t]{2}{*}{2} & $\begin{array}{l}\text { Agara Lake } \\
\text { G. scripta (L.) Ach. }\end{array}$ & 35.59 & 203.10 & 863 & 384.55 & 10.06 & 600.76 \\
\hline & leucosorodes Nyl. & 3.04 & 149.15 & 570 & 79.86 & 5.84 & 460.01 \\
\hline 3 & $\begin{array}{l}\text { Madiwala Market } \\
P \text {. cocoes (Swartz) Nyl. }\end{array}$ & 10.31 & 101.4 & 9795 & 224.6 & 19.32 & 600.54 \\
\hline 4 & $\begin{array}{l}\text { Ayappa Swamy Temple Road } \\
\text { D. aegialita (Afz. in Ach.) } \\
\text { Moore }\end{array}$ & ND & ND & 7358 & 322.39 & 16.06 & 720.34 \\
\hline 5 & $\begin{array}{l}\text { Madiwala Police Station } \\
\text { Junction } \\
\text { L. perplexa } \text { Brodo }\end{array}$ & 7.96 & 199.32 & 265 & 531.5 & 7.37 & 644.10 \\
\hline 6 & $\begin{array}{l}\text { Bomannahalli traffic junction } \\
* P \text {. austrosinensis (Zahlbr.) Hale }\end{array}$ & 34.57 & 623.95 & 4530 & 553.92 & 158.32 & 703.12 \\
\hline 7 & $\begin{array}{l}\text { Begur Road** P. nanospora (A. } \\
\text { Singh) Upreti }\end{array}$ & 36.43 & 175.9 & 1506 & 831.01 & 18.28 & 698.13 \\
\hline 8 & $\begin{array}{l}\text { Mangamanapalya Road } \\
\text { C. candelaris }(\text { L.) Laundon }\end{array}$ & 13.79 & 484.69 & 748 & 103.82 & 19.66 & 650.23 \\
\hline 9 & $\begin{array}{l}\text { B.D.A Complex H.S.R Layout } \\
\text { D. aegialita (Afz. in Ach.) } \\
\text { Moore }\end{array}$ & ND & 46.4 & 6887 & 98.6 & 8.99 & 351.67 \\
\hline 10 & $\begin{array}{l}\text { Chennakeshavanagar Main Road } \\
* * * \text { P. petricola Nyl. In Crombie }\end{array}$ & 95.29 & 183.33 & 9202 & 133.05 & 115.19 & 800.73 \\
\hline 11 & $\begin{array}{l}\text { Shanthi Niketan gardens MICO } \\
\text { Naganathapura plant**** } \\
\text { P. petricola Nyl. in Crombie }\end{array}$ & 18.47 & ND & 5538 & 105.38 & 338.12 & 1409.34 \\
\hline 12 & $\begin{array}{l}\text { Central prison Road } \\
\text { C. candelaris }(\mathrm{L} .) \text { Laundon }\end{array}$ & 13.79 & 84.69 & 748 & 103.82 & 19.66 & 843.10 \\
\hline 13 & $\begin{array}{l}\text { Infosys garden Electronic City } \\
\text { C. candelaris (L.) Laundon }\end{array}$ & 19.00 & ND & 6926 & 157.496 & 19.75 & 343 \\
\hline 14 & $\begin{array}{l}\text { wipro Garden Electronic City } \\
\text { P. praesorediosa (Nyl.) Hale }\end{array}$ & 28.23 & 164.35 & 2040 & 126.85 & 15.65 & 390 \\
\hline 15 & $\begin{array}{c}\text { Electronic city Phase } 1 \text { traffic } \\
\text { junction. } \\
P . \text { concinna Erichsen }\end{array}$ & 2.50 & ND & 55.47 & 351.68 & 5.02 & 690.01 \\
\hline
\end{tabular}


Table 4. Heavy metal concentrations $/ \mu \mathrm{g} \mathrm{g}^{-1}$ dry weight in soils of Hosur Road, Bangalore South.

\begin{tabular}{|c|c|c|c|c|c|c|c|c|c|c|c|c|c|}
\hline \multirow[b]{2}{*}{ Stations } & \multirow[b]{2}{*}{$\begin{array}{c}\text { Name of the Sample locations } \\
\text { in Hosur Road }\end{array}$} & \multicolumn{2}{|c|}{$\mathrm{Cr}$} & \multicolumn{2}{|c|}{$\mathrm{Pb}$} & \multicolumn{2}{|c|}{$\mathrm{Fe}$} & \multicolumn{2}{|c|}{$\mathrm{Zn}$} & \multicolumn{2}{|c|}{$\mathrm{Cu}$} & \multicolumn{2}{|c|}{$\mathrm{Ni}$} \\
\hline & & 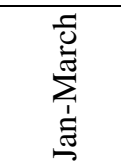 & $\begin{array}{l}\text { z } \\
z_{1} \\
\stackrel{0}{0} \\
0 \\
0\end{array}$ & 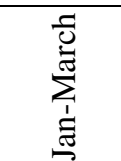 & 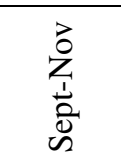 & 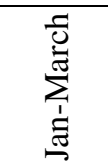 & $\begin{array}{l}z \\
z \\
\vdots \\
0 \\
0 \\
0\end{array}$ & 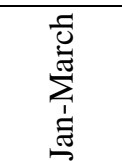 & $\begin{array}{l}\overrightarrow{0} \\
z \\
\vdots \\
0 \\
0 \\
0\end{array}$ & 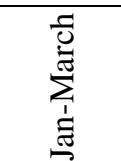 & 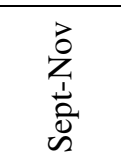 & 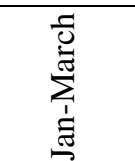 & 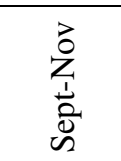 \\
\hline 1 & Kendriva Sadan Koramangala & ND & ND & ND & ND & 748 & 235 & 49.34 & 24.34 & 17.34 & 9.43 & 420.90 & 350 \\
\hline 2 & Agara Lake & 88.56 & 56.33 & 234.56 & 146.35 & 7242 & 6899 & 235.16 & 163.78 & 42.17 & 23.49 & 453.70 & 345.67 \\
\hline 3 & Madiwala Market & 54.34 & 49.43 & 226.43 & 178.23 & 4533 & 3456 & 435.34 & 345.15 & 13.54 & 9.45 & 600.76 & 454.25 \\
\hline 4 & Ayappa Swamy Temple Road & 52.23 & 44.67 & 124.34 & 56.43 & 2530 & 1229 & 234.19 & 167.44 & 12.30 & 10.34 & 460.01 & 346.60 \\
\hline 5 & $\begin{array}{l}\text { Madiwala Police Station } \\
\text { Junction }\end{array}$ & 66.31 & 49.45 & 127.41 & 98.24 & 12400 & 6784 & 275.14 & 201.54 & 134.28 & 98.34 & 600.54 & 543.50 \\
\hline 6 & Bomannahalli traffic junction & 145.45 & 79.45 & 298.34 & 94.24 & 11400 & 7432 & 423.66 & 198.57 & 25.43 & 20.66 & 720.34 & 600 \\
\hline 7 & Begur Road & 123.67 & 65.87 & 279.54 & 200.46 & 976 & 654 & 435.15 & 345.44 & 11.37 & 9.23 & 644.10 & 544.65 \\
\hline 8 & Mangamanapalya Road & 56.62 & 50.23 & 623.95 & 423.87 & 4630 & 3324 & 534.19 & 450.23 & 162.85 & 98.43 & 703.12 & 573.25 \\
\hline 9 & B.D.A Complex H.S.R Layout & ND & ND & 134.52 & 56.23 & 576 & 560 & 111.23 & 78.45 & 15.67 & 13.34 & 698.13 & 432.34 \\
\hline 10 & $\begin{array}{l}\text { Chennakeshavanagar Main } \\
\text { Road }\end{array}$ & 72.56 & 56.71 & 484.69 & 305.60 & 16234 & 12965 & 234.51 & 164.23 & 23.45 & 12.46 & 650.23 & 540.28 \\
\hline 11 & $\begin{array}{l}\text { Shanthi Niketan Gardens } \\
\text { MICO Naganathapura plant }\end{array}$ & ND & ND & 41.39 & 23.56 & 4847 & 3671 & 59.34 & 44.11 & 198.23 & 145.34 & 1409.34 & 663.50 \\
\hline 12 & Central Prison Road & 33.34 & 13.63 & 183.33 & 126.73 & 6820 & 4569 & 156.56 & 68.65 & 115.19 & 67.45 & 800.73 & 604.51 \\
\hline 13 & Infosys garden Electronic City & 67.45 & 47.98 & ND & ND & 4984 & 3421 & 93.23 & 58.26 & 5.34 & 1.29 & 351.67 & 20.45 \\
\hline 14 & wipro Garden Electronic City & 58.89 & 43.21 & ND & ND & 655 & 355 & 127.12 & 59.23 & 6.89 & 3.46 & 843.10 & 450.00 \\
\hline 15 & $\begin{array}{l}\text { Electronic city Phase } 1 \\
\text { Junction. }\end{array}$ & 134.67 & 79.45 & 345.56 & 244.63 & 7234 & 6234 & 393.28 & 198.58 & 22.51 & 18.45 & 343 & 20.45 \\
\hline
\end{tabular}




\section{Conclusions}

Monitoring of heavy metals through litchen species provides efficient way to assess the qualitative and quantitative differences in metal concentrations at distinct locations and on local and landscape scales. Under the present ecological conditions the heavy metal load is significant in Hosur Road, Bangalore.

\section{Reference}

1. Karnataka State Pollution Control Board, Water quality monitoring of lakes in and around Bangalore city, Bangalore, 2001, 1, p. 139.

2. www.http://parisara.kar.nic.in/lda.htm as accessed on 13 September 2006.

3. Bangalore Metropolitan Rapid Transport Limited, Environmental Impact Analysis, 2006

4. http://en.wikipedia.org/wiki/Bangalore_Metropolitan_Environment

5. Ming Chen and Ma, Lena Q, Soil Sci Soc Am J., 2001, 65, 491-499.

6. Lark B S, Mahajan R K and Walia T P S, Indian J Environ Health., 2002, 44, 164-167.

7. Awasthi D D, Lichenology in Indian Subcontinent, Bishen Singh Mahendra Pal Singh, Dehradun, India, 2000, p. 124.

8. Richardson D H S, Pollution Monitoring with Lichens, Richmond Publishing Co Ltd, England, 1992, p. 76.

9. Rajesh Kumar Sharma, Madhoolika Agrawal and Marshall F M, Effects of waste water irrigation on heavy metal accumulation in soil and plants. Paper presented at a National Seminar, Bangalore University, Bangalore, 2004, Abst. No. 7, p. 8.

10. Fernandes H M, Bidene E D, Veiga C H S and Patchinncelan S R, Environ Pollut., 1994, 85, 259-64.

11. Laxen D and Harrison R C, Water Res., 1997, 11, 1-11. 


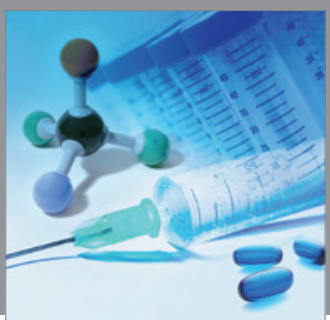

International Journal of

Medicinal Chemistry

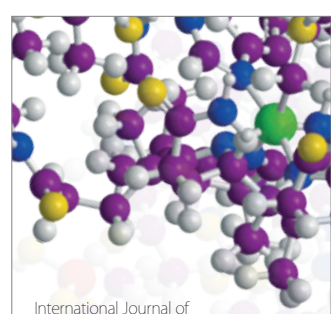

Carbohydrate Chemistry

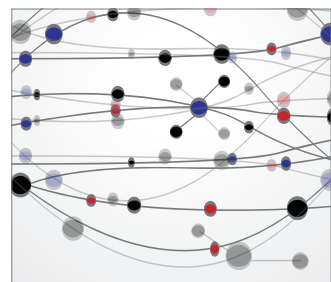

The Scientific World Journal
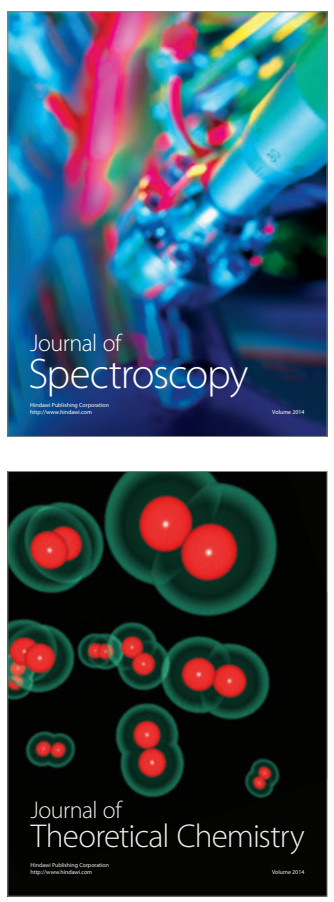
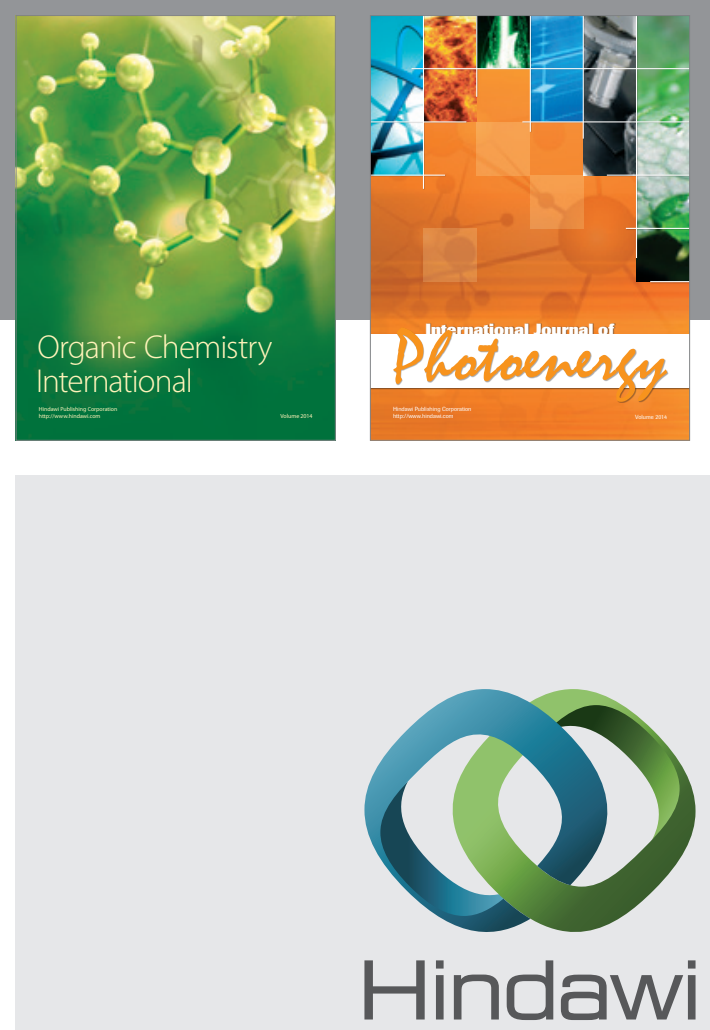

Submit your manuscripts at

http://www.hindawi.com
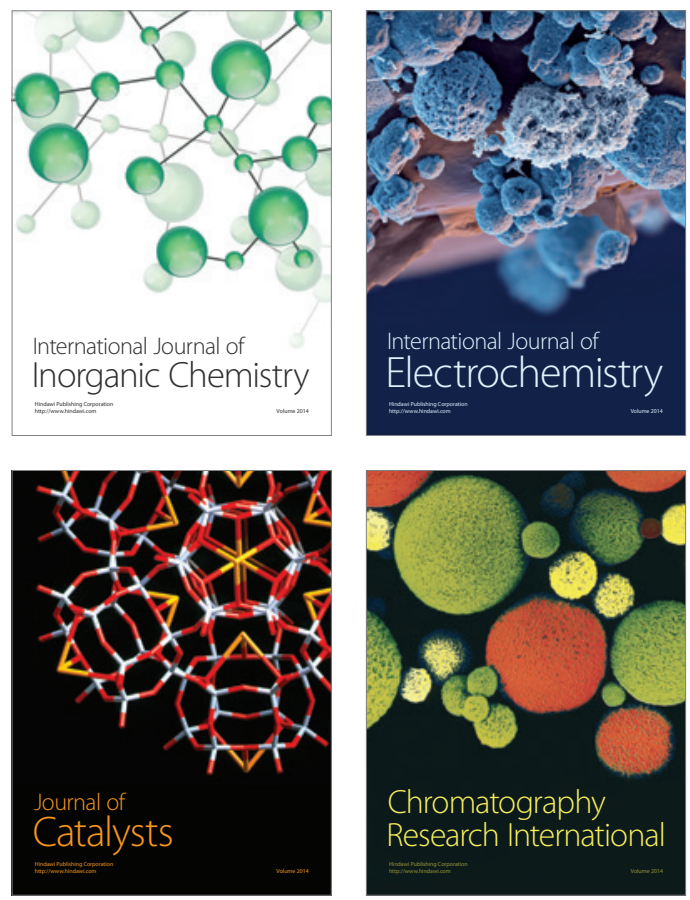
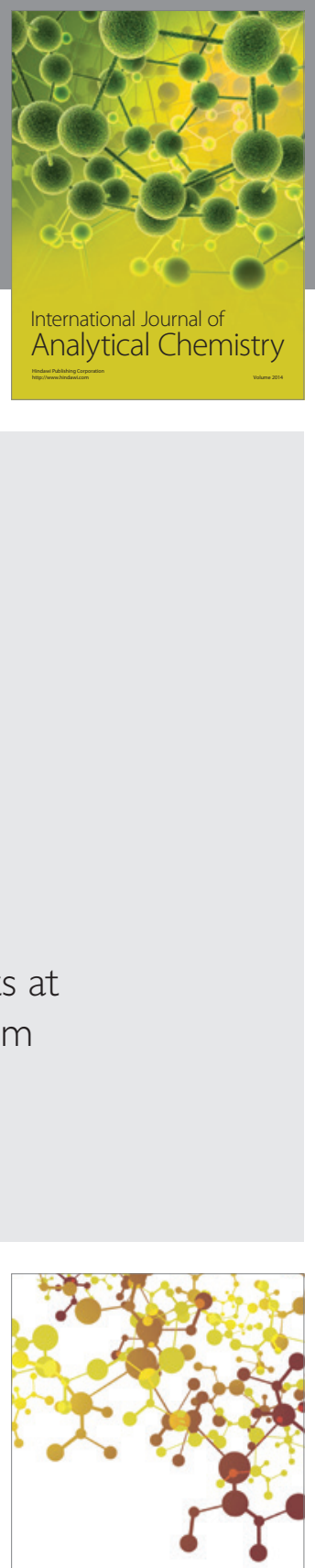

Journal of

Applied Chemistry
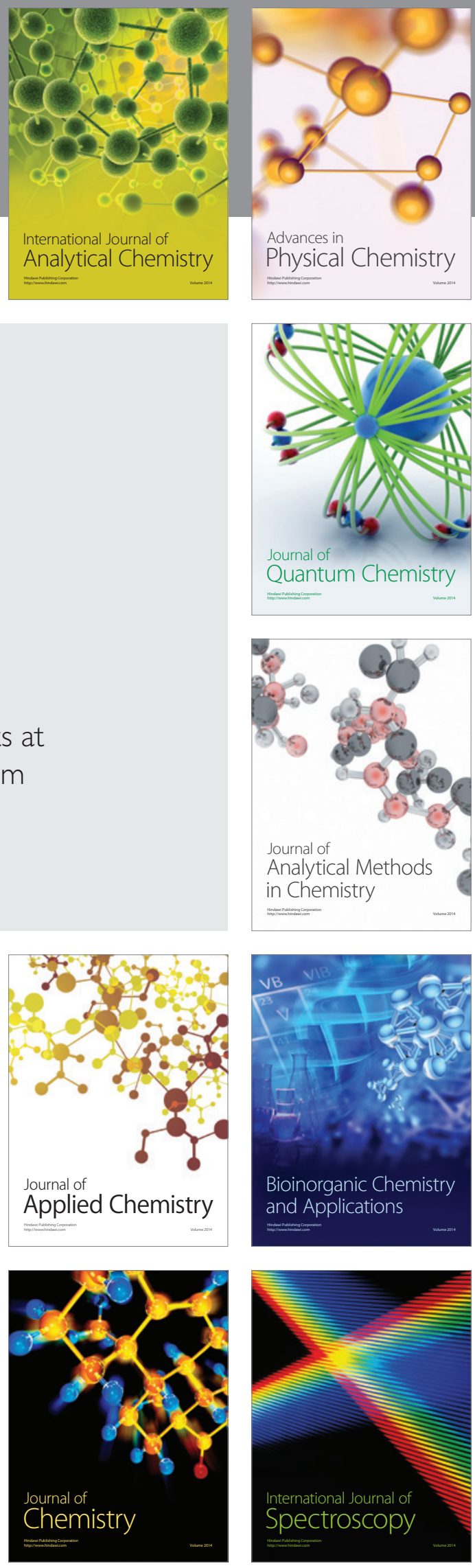